\title{
12 Lead Placement Standard
}

National Cancer Institute

\section{Source}

National Cancer Institute. 12 Lead Placement Standard. NCI Thesaurus. Code C71102.

An electrocardiogram (ECG) lead placement whereby 12 leads are recorded, with each lead representing an electrical "view" of the heart. The six leads recorded in the frontal plane are derived from the placement of 3 electrodes (RA or Right Arm, LA, or Left Arm, and LL or Left Leg). These bipolar frontal leads form the basis of Einthoven's triangle, and are represented by leads I, II, and III. Three other derived (or augmented) bipolar frontal vectors are also recorded on a standard 12-lead EKG, aVR, aVF, and aVL. 6 unipolar leads, corresponding to V1 - V6 measure the electrical activity in the horizontal plane. The placement for the "V" leads is as follows: V1: right 4th intercostal space,V2: left 4th intercostal space, V3: halfway between V2 and V4, V4: left 5th intercostal space, midclavicular line, V5: horizontal to V4, anterior axillary line, V6: horizontal to V5, mid-axillary line. 